\title{
Impactos de los créditos agropecuarios de Financiera Nacional de Desarrollo en el bienestar de los hogares beneficiarios del valle de Mexicali
}

\author{
Zaira González Rodríguez*, Juan Antonio Leos Rodríguez ${ }^{* *}$ y María Jesica Zavala Pineda**
}

Recepción: 15 de febrero de 2016

Aceptación: 5 de mayo de 2016

*Universidad Autónoma Chapingo, México.

${ }^{* *}$ Centro de Investigaciones Económicas, Sociales

y Tecnológicas de la Agroindustria y la Agricultura Mundial, México.

Correos electrónicos: gonzalez.r.zaira@gmail.com; jleos45@gmail.com; jesikzp@gmail.com

Se agradecen los comentarios de los árbitros de la revista.

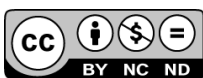

Resumen. Por medio del método de pareamiento por puntaje de propensión, se analiza y cuantifica el impacto sobre el bienestar que los créditos otorgados al sector agroindustrial y rural por Financiera Nacional de Desarrollo (FND) tienen en las familias beneficiarias del valle de Mexicali, en el estado de Baja California. Para obtener los datos, se elaboró una encuesta con preguntas sobre la inversión, alimentación, educación y salud de las familias. FND espera que los proyectos productivos para los que se otorgó el crédito creen un efecto multiplicador en el ingreso de las familias permitiendo una mejora en su calidad de vida. Los principales resultados muestran un impacto positivo en los alimentos que son bienes normales, adquisición de tractores, gasto educativo y visitas al doctor. Palabras clave: crédito rural, crédito agrícola, financiera nacional de desarrollo, agricultura, impacto social, bienestar, pobreza, rural, banca de desarrollo.

\begin{abstract}
Agricultural Credit Impacts from FND (Financiera Nacional de Desarrollo) on the Welfare of Beneficiary Households in the Valley of Mexicali

Abstract. This paper analyzes the impact that credits from Financiera Nacional de Desarrollo (FND) aimed at rural and agricultural projects had on the welfare of families from the Valley of Mexicali, in the state of Baja California, Mexico. The logic behind the program is that credit will be invested in productive projects, creating a multiplier effect in the families' income and allowing them to improve their quality of life. This research focused on the credits' impact on participating families through a Propensity Score Matching methodology. To obtain the necessary data for this analysis, a survey was constructed with questions on investment, food consumption, education, and health of the participating families. The main results showed a positive impact on food consumption of normal goods, as well as on education expenditure, ownership of a tractor, and doctor visits.
\end{abstract}

Key words: Rural Credit, Agricultural Credit, Financiera Nacional de Desarrollo, Agriculture, Social Impact, Welfare, Poverty, Rural, Development Bank.

\section{Introducción}

Como parte de una estrategia para activar la economía de áreas primordialmente agrícolas, el gobierno mexicano provee de apoyos crediticios a zonas rurales por medio de Financiera Nacional de Desarrollo Agropecuario, Rural, Forestal y Pesquero (FND). Esta banca de desarrollo comienza sus operaciones en 2003 con la misión de "desarrollar al medio rural a través de financiamiento en primer y segundo piso para cualquier actividad económica que se realice en poblaciones menores a 50000 habitantes resultando en la mejora de su calidad de vida" (FND, 2015). Para lograr su misión, FND brinda créditos y asesorías de manera directa e indirecta para el desarrollo de actividades agropecuarias, forestales y pesqueras, entre otras. 
Este trabajo indaga en el impacto que tienen las transferencias de FND en el bienestar de las familias participantes del valle de Mexicali, Baja California. La literatura del impacto de la FND en específico es inexistente, a pesar de esto existe información del impacto que el cambio de Banrural a la Financiera Rural en 2003 y a la FND en el 2014 tuvo en el mercado crediticio nacional en zonas rurales agropecuarias. Pérez et al. (2009) documentó la contracción del crédito agrícola en el sur de Sonora, pues tanto las garantías hipotecarias y líquidas para acceder al crédito agrícola, la cantidad del crédito de las fuentes financieras, así como la falta de financiamiento para proyectos de tecnificación de los cultivos se volvieron insuficientes con el nuevo modelo de servicios financieros.

Sin embargo, este cambio también trajo consigo un manejo adecuado de las tasas de interés que garanticen el cumplimiento del pago del crédito, además de que las herramientas de la Financiera han resultado tan eficientes que intermediarios financieros rurales y dispersoras de crédito han solicitado su apoyo y capacitación para incrementar su eficiencia en la colocación y recuperación de recursos (Soto Ibáñez, 2008).

En lo que respecta al nivel de vida, resulta difícil calcularlo por medio del ingreso, que es la variable más usada para medir el bienestar de un hogar debido a las variaciones en los ingresos de los agricultores y a la dificultad que tienen de discernir la ganancia que queda después de recibir y pagar el crédito, además de ser una variable endógena a diferentes proxys de bienestar. Por ello, se analiza el impacto de FND en la alimentación, inversión, educación y salud de las familias por medio del método de Pareamiento por Puntaje de Propensión (PPP). Se espera observar un impacto positivo en todas las variables mencionadas, con excepción de aquellos alimentos que califican como bienes inferiores.

Existe una plétora de estudios que utilizan estas cuatro variables para medir el bienestar de programas de crédito. En lo que concierne a alimentación, para examinar el impacto de Progresa y Procampo, Ruiz-Arranz (2002) se enfoca en la diferencia de impacto entre un programa dirigido al campo y otro dirigido al consumo y encuentra que ambos programas aumentan el consumo de carne y vegetales. Meng et al. (2009) utiliza el consumo de calorías para evaluar la relación que el rápido crecimiento económico y las reformas en China tuvieron en la década de los noventa y descubre que el consumo calórico bajó durante estos años debido a que se redujo la participación del Estado en la provisión de bienes y servicios públicos.

Otro impacto que se ha estudiado en la literatura del crédito y desarrollo es la educación y la salud. En su estudio,
Crépon et al. (2011) encontró evidencia de pequeños aumentos en el gasto en educación y salud por la disponibilidad de microcréditos. La mejora en la escolarización de los niños es también muy pequeña; sin embargo, la disponibilidad de microcréditos no se encontró relacionada a un aumento en el trabajo infantil (que podría ser un potencial efecto negativo dado que estos créditos son usados para la inversión en empresas familiares). Otro dato relevante es que los hogares de tratamiento son más propensos a gastar en la salud y menos en eventos sociales. Pitt et al. (2003) se centró en el impacto que los microcréditos para mujeres tienen en la salud de los niños usando su altura y la circunferencia de sus brazos como proxys. Se encontró efectivamente que estos microcréditos tienen un impacto significativo.

\section{Materiales y métodos}

Los datos para este trabajo se obtuvieron por medio de una encuesta creada precisamente para fines de esta investigación. Como base se utilizaron preguntas de la Encuesta Nacional sobre Niveles de Vida de los Hogares Mexicanos 2009, la cual es parte de un proyecto para proporcionar información de las "dimensiones del bienestar de la población mexicana y estudiar los cambios en dicho bienestar a través del tiempo (ENNviH, 2012). Además del formato de las preguntas, los datos de esta encuesta fueron también utilizados para realizar el ppp de las observaciones de Mexicali.

La técnica del ppp es utilizada para estimar el impacto de los créditos en el bienestar de las familias encuestadas, pues en el caso que nos ocupa no existe una selección aleatoria de las observaciones de tratamiento y da paso a la condición de sesgo de selección. El análisis de ppp comienza con un t-test para buscar diferencias estadísticas entre las covariables de los grupos de control y de tratamiento. Después se construye un grupo de control para compararlo con el grupo de tratamiento a través de un modelo de probabilidad de participación usando características observables que influyan en el programa, por medio de un modelo discreto; para esta investigación se utilizó el modelo de probit. Cabe mencionar que al utilizar el modelo de logit, los resultados del pareamiento fueron idénticos, ya que ambos son modelos binarios (Caliendo y Kopeinig, 2005).

Aprovechando el modelo de probit, cada una de las observaciones en el grupo de tratamiento es "emparejada" con una observación del grupo de control con un puntaje de propensión similar. Lo anterior permite el cálculo del Impacto del Tratamiento sobre los Tratados (ATT, por sus siglas en inglés). El método utilizado en esta investigación 
es el emparejamiento nearest-neighbor, en el cual cada una de las observaciones del grupo de tratamiento es emparejada con una o más de las del grupo de control que estén más próximas a su valor.

La validez del ppp depende de dos supuestos en los datos: a) la independencia condicional y b) la existencia de soporte común. La independencia condicional significa que los factores no-observables no afectan la participación en el programa. Para que se cumpla este supuesto es importante una cuidadosa selección de las variables que conformarán el vector de variables que impactan la probabilidad de obtener un crédito de FND, de otra manera la validez del PPP se vuelve cuestionable (De Janvry et al., 2011). La segunda condición es la de la existencia de soporte en común, o traslape en los puntajes de propensión, entre los individuos participantes y los no participantes en el tratamiento. Para la estimación del ATT, este supuesto puede ser descrito como:

$P\left(T_{i}=1 \mid X_{i}\right)<1$

donde las características observables que no son afectadas por el tratamiento (covariables) son representadas por $X$ y el tratamiento por $T$.

El impacto del tratamiento para cualquiera de los cuatro enfoques de bienestar usados en esta investigación (alimentación, inversión, educación y salud) para un individuo $i$ puede ser definido como:

$\tau_{i}=Y_{i}(1)-Y_{i}(0)$

donde el indicador de tratamiento $D_{i}$ es igual a 1 si el individuo $i$ recibe el tratamiento (en este caso crédito de FND) y 0 si no. Los resultados posibles son definidos como $Y_{i}\left(D_{i}\right)$ para cada individuo $i$, donde $i=1, \ldots, N$ y $N$ denota el total de la población. Sin embargo, solamente se observa un sólo resultado, ya sea $Y_{i}(1)$ o $Y_{i}(0)$ para cada individuo $i$. Al otro resultado no observable se le denomina como el contrafactual. Por lo tanto, estimar $\tau_{i}$ se vuelve imposible y es necesario cambiar la investigación hacia los efectos de tratamiento promedios en la población. Así, el objetivo es obtener la siguiente ecuación:

$\tau A T T=E(\tau \mid D=1)=E[Y(1) \mid D=1]-E[Y(0) \mid D=1]$

La media del contrafactual para las observaciones con tratamiento en la población se representa como $E[Y(0) \mid D$ $=1]$, que al ser no observable se tiene que sustituir para poder estimar el ATT. El uso de la media de individuos sin tratamiento, $E[Y(0) \mid D=0]$ no puede ser utilizada como sustituto debido a problemas de sesgo de selección. Sin embargo, con la existencia de soporte en común se puede obtener el estimador PPP para el ATT, que se puede escribir de la siguiente manera:

$$
\begin{aligned}
\tau P S M A T T= & E P(X) \mid D= \\
& 1\{E[Y(1) \mid D=1, P(X)]-E[Y(0) \mid D=0, P(X)]\},
\end{aligned}
$$

donde $X$ representa la lista de covariables y el puntaje de propensión es $P(X)$. En otras palabras, el estimador pPp es simplemente la diferencia media en los resultados de impacto en el área de soporte común (Caliendo y Kopeinig, 2005).

Ya que el método de pareamiento se basa en el supuesto de independencia condicional, en la literatura reciente (Azam et al., 2013; Clément, 2011; DiPrete y Gangl, 2004) se utiliza el análisis de sensibilidad para estimar la sensibilidad de los resultados respecto a desviaciones de este supuesto. En esta investigación se usó el método de límites de Rosenbaum (2002) con el objetivo de determinar si "el efecto promedio en el tratamiento puede ser modificado por variables no observadas, creando un sesgo escondido" (Clément, 2011: 16). En este trabajo se reporta el valor de $p$ del límite superior de la hipótesis nula "efecto de tratamiento promedio es cero" para diferentes valores de $\Gamma$. Si para los intervalos de $\Gamma$ menores a 2 el valor de $p$ de la hipótesis nula es menor a 0.05 , entonces se afirma que el efecto de tratamiento es robusto a la presencia de sesgo escondido (Clément, 2011).

\section{Resultados y discusión}

\section{1. Impacto en el gasto en alimentos}

El análisis del impacto en el bienestar de FND comienza con un t-test (Anexo A1, lista A) para buscar diferencias estadísticas entre las covariables de los grupos beneficiarios y los no beneficiarios. La hipótesis nula que establece que ambos grupos tienen las mismas características se rechaza para 17 de las 22 variables con un nivel de confianza de 95\%. Después se construye un modelo de probit para calibrar el puntaje de propensión en la muestra de individuos encuestados (anexo A2, lista A), el cual muestra una pseudo $R^{2}$ de 0.5833 (anexo A2, lista A). El poder explicativo del modelo es relativamente alto, lo que sugiere que los dos grupos de individuos son diferentes en muchas de sus características (Azam et al., 2013). La significancia estadística del modelo de probit no se discute, ya que la ecuación de participación no es un modelo de determinantes; por lo tanto, esta información no es relevante para el objetivo del presente estudio. 
El impacto en el consumo de alimentos se midió como el total y como el porcentaje del gasto total en alimentos per cápita, pero también en gasto por las categorías de carne, tortillas, frijoles, frutas y vegetales. Para todos los análisis de PPP de gasto total por categoría (anexo A1, lista A) las observaciones de los grupos de control y de tratamiento son las mismas, donde las observaciones del soporte común están constituidas por 34 observaciones de tratamiento y 1235 de control. Por otro lado, el soporte común del pareamiento para el porcentaje de gasto por categoría de alimento (anexo A2, lista A) está constituido por 27 observaciones de tratamiento y 1225 de control. La distribución de pareamiento para las variables dependientes "porcentaje de gasto en refrescos" y "porcentaje de gasto en tortillas" corresponde a las figuras y del Anexo A1, lista A. La gráfica 1 muestra el traslape en la frecuencia de la distribución del puntaje de propensión para las observaciones con un puntaje por encima de 0.1 correspondientes a esta sección (el resto de las observaciones cuenta con un puntaje de propensión por debajo de 0.1). Si bien el traslape no es perfecto, resulta suficiente para este trabajo.

El desempeño del traslape se refleja en el t-test del anexo A3 realizado después del pareamiento: no se puede rechazar la hipótesis nula de que ambos grupos tienen las mismas características más que para la variable "número de cuartos usados para dormir", pero esta diferencia resulta no significativa en un intervalo de confianza de 99\%. El indicador $p>$ chi $^{2}$ es de 0.342 para la lista A1 del anexo A3 y 0.490 para la lista A del anexo A2. Este indicador representa la probabilidad de obtener el chi $^{2}$ (24.10) si la hipótesis nula es verdadera, la cual dice que todas estas variables juntas no tienen impacto en la participación en FND. Dado el valor de $p>\mathrm{chi}^{2}$, no se puede rechazar la hipótesis nula, que era lo que se buscaba con el ppr. Lo mismo aplica para los resultados de la lista A del anexo A2.

Gráfica Traslape en la frecuencia de la distribución del puntaje de propensión por encima de 0.1 de las observaciones para estimaciones de gasto en alimentos.

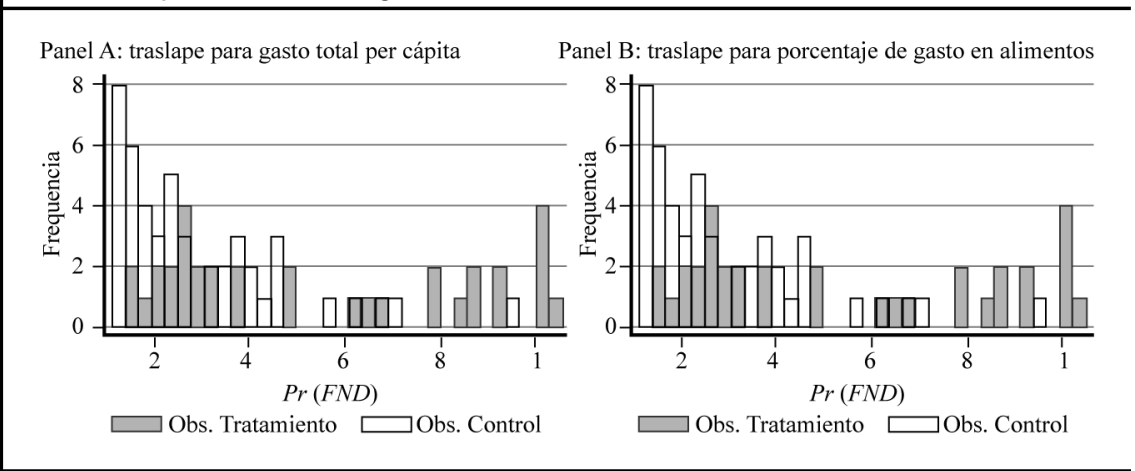

Nota: $P r=$ probabilidad

Fuente: elaboración propia con datos obtenidos de la encuesta realizada para la presente investigación y ENNviH (2012).
Los resultados en el anexo A4 (lista A1) muestran que existe un impacto positivo en todas las variables de consumo a un nivel de intervalo de confianza de $99 \%$, excepto en el caso de bienes inferiores, pues en esta variable el impacto es positivo a un nivel de 95\%. Este último impacto resulta el de menor magnitud (17 pesos), mientras que las diferencias más notorias entre el grupo de tratamiento y el de control se encuentran en las categorías de frutas y vegetales y en gasto en alimentos industrializados per cápita, donde el grupo de tratamiento gasta 490\% y 475\% más que el grupo de control. La lista A2 del anexo A4 muestra el impacto de participación en el programa en el porcentaje de gasto por categorías de alimento: es positivo para todas las categorías; sin embargo, no es significativo para bienes inferiores y para tortillas. La lista A2 del anexo A4 muestra un impacto relativamente más fuerte para las categorías de carne y de frutas y vegetales, donde el grupo de tratamiento muestra un porcentaje de consumo por encima del grupo de control de un 14 y $15 \%$, respectivamente.

Además de calcular el impacto de los créditos en el gasto por medio del método de pareamiento nearest-neighbor, cinco métodos de pareamiento alternativo son calculados en el anexo A5 para comparar y determinar si estos arrojarían resultados significativamente diferentes: caliper de 0.01 y 0.001 , kernel con un tamaño de banda de 0.2 y 0.02, 5 nearest-neighbor matching y pareamiento sin repuesto. No obstante, los resultados de estos métodos alternativos fueron similares a los reportados en el anexo A4. Cabe destacar que el tamaño de la banda kernel y el método caliper para indicar el nivel de tolerancia para la distancia máxima que puede haber entre los pareamientos por puntaje utilizados en este análisis fueron escogidos de acuerdo con literatura existente (Azam et al., 2013; Dehejia y Wahba, 2002; Liu y Lynch, 2011). El impacto en la lista A2 del anexo A4 muestra que el impacto resulta no significativo para las categorías de bienes inferiores y tortillas.

\section{2. Impacto en inversión}

Para evaluar el impacto en la inversión, las proxys de posesión de tractor y posesión de piso de madera o loseta en la vivienda son usadas. Después de realizar el pareamiento las observaciones emparejadas resultaron ser las mismas que las que aparecen en el anexo A1, lista A (34 observaciones de tratamiento y 1235 observaciones de control), por lo que las figuras de traslape y el t-test después del pareamiento son idénticos a los presentados para la sección pasada. 
El anexo A6 muestra el ATT de FND en las diferentes proxys para inversión. La variable de posesión de tractor es impactada de manera significativa, mientras que la variable de posesión de piso de madera o loseta en la vivienda muestra un error estándar que vuelve el resultado del impacto no significativo. Ambos resultados son respaldados por los métodos alternativos de pareamiento del anexo A7: todos los resultados para posesión de tractor son positivos y estadísticamente significativos (salvo al usar caliper de 0.001), mientras que los resultados de impacto en la posesión de piso de madera o loseta no lo son.

\section{3. Impacto en los gastos educativos}

Una lista de covariables $X$ diferente de la sección de alimentación y la de inversión fue utilizada para realizar un t-testinicial (anexo A1, lista B) y un modelo de probit (anexo A2, lista B) para el análisis de impacto en gastos educacionales. El modelo de probit usado para obtener el puntaje de propensión para participación en el programa muestra una $R^{2}$ relativamente alta con 0.4921 .

Las primeras dos proxys de educación examinadas en esta sección son las de gasto total en educación y gasto en educación excluyendo el transporte. El número de observaciones del grupo de tratamiento emparejadas para estos dos análisis es de 700 observaciones de control y 26 de tratamiento. Esta cifra baja se debe en parte a que no en todas las familias existen individuos que asisten a la escuela. Adicionalmente, dado que el impacto de participación en el programa es calculado por gasto educativo, existe mucha varianza en las observaciones, pues los altos niveles educacionales, como el universitario, implica mayores gastos comparado con los de preescolar o primaria. El éxito del pareamiento puede ser verificado en la gráfica 1 , mientras que el t-test después del matcbing en el anexo A8 (lista B1) no muestra diferencia después del pareamiento para ninguna de las variables, además de que la hipótesis nula no puede ser rechazada (pseudo $R^{2}$ es 0.332). Al analizar el ATT del programa tanto en el gasto total educativo por estudiante como en el gasto educativo por estudiante excluyendo gastos de transporte, la estadística $t$ indica que el impacto fue significativo (anexo A9). Estos resultados se mantienen al usar métodos alternativos de pareamiento (anexo A10).

En el caso de la tercera proxy de impacto del programa en educación "gasto de transporte escolar por estu- diante", el $t$-test realizado después del pareamiento del anexo A8 lista B2 tampoco mostró diferencias entre la media del grupo de tratamiento y la del grupo de control. Para este análisis el grupo de tratamiento con traslape fue de solo 22 observaciones, mientras que el grupo de control estuvo constituido por 700 , mostrando una diferencia de gasto de 1058 pesos anuales.

La gráfica 2 muestra el éxito del traslape. A pesar de que el traslape no es perfecto, el impacto de programa en esta categoría es significativo con una estadística $t$ de 3.06 (anexo A9). Este resultado es también respaldado por los resultados del anexo A10, donde cuatro de los cinco métodos de pareamiento alternativos muestran un impacto de participación en el programa significativo similar al del anexo A9.

\section{4. Impacto en salud}

Las variables y las observaciones emparejadas en esta sección son las mismas que las correspondientes para el modelo de probit y el $t$-test del anexo A2 y A3, lista A y A1 respectivamente. El ATT de FND en las seis diferentes medidas de salud en términos totales y porcentuales se muestra en el anexo A11. Estos resultados tienen los valores que se esperaban: para el análisis de totales (anexo A11, izquierda), significativos y negativos para "número de individuos que enfermaron y no visitaron un doctor" y para "número de individuos que enfermaron", y significativos y positivo para "número de individuos que no enfermaron y visitaron un doctor". Esta última variable puede ser interpretada como el impacto de los créditos en revisiones preventivas como lo son las visitas al doctor debido a condiciones de salud permanentes, como la diabetes. En cuanto al análisis de porcentajes (anexo A11, derecha), los impactos tienen los mismos signos que los impactos del análisis de totales (anexo A11, izquierda), mas la diferencia es que todas las variables resultaron tener impactos
Gráfica 2. Traslape en la frecuencia de la distribución del puntaje de propensión por encima de 0.1 de las observaciones para estimaciones de gasto en educación.

Panel A: traslape para gasto en educación y exclusión de Panel B: traslape para gasto en transporte escolar gastos de transporte

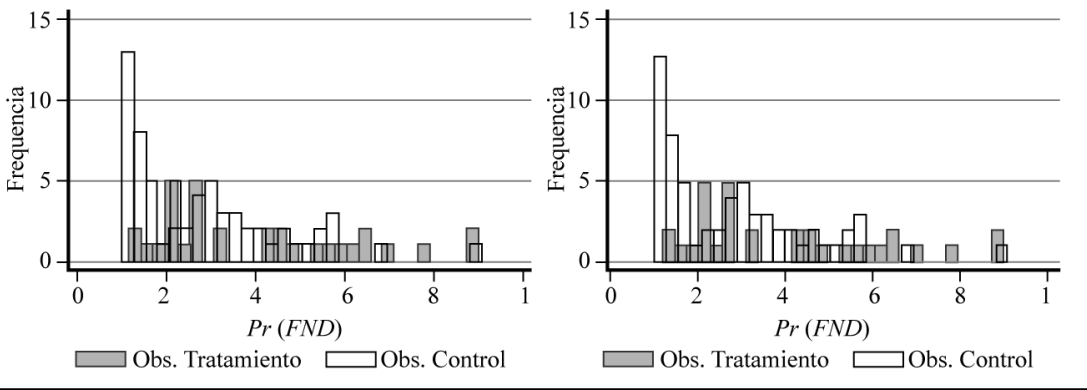

ota: $\operatorname{Pr}=$ probabilidad

Fuente: elaboración propia con datos obtenidos de la encuesta realizada para la presente investigación y ENNviH (2012). 
significativos, salvo la variable "porcentaje de individuos que enfermaron y visitaron un doctor".

Aunque el impacto en la cuarta y sexta variable del anexo A11, "número de individuos que no enfermaron y no visitaron un doctor" y "número de individuos que visitaron un doctor" resultó no significativo bajo el método de pareamiento de nearest matching, el uso de métodos alternativos de pareamiento (anexo A12) arrojó resultados con una estadística $T$ por encima de 2.021, lo que indica un impacto positivo. En lo que concierne al resto de los métodos alternativos de pareamiento, estos mostraron el mismo signo de impacto de los créditos y la misma significancia o falta de ella.

\section{5. Análisis de sensibilidad}

Se realizó un análisis de sensibilidad a la presencia de variables relevantes no medidas para los pareamientos por puntaje de propensión realizados en este trabajo utilizando el comando de rbounds del software de Stata. Se calcularon los límites de Rosenbaum para el АтT en presencia de heterogeneidad no observada entre el grupo de tratamiento y el de control. El anexo A13 muestra el valor de gamma $(\Gamma)$ y niveles de significancia de los límites superiores. Estos últimos son los valores $P$ de la hipótesis nula de impacto de tratamiento $=$ 0 , siendo la alternativa $>0$. Gamma representa el supuesto de "heterogeneidad o endogeneidad no medida en la asignación del tratamiento expresado en términos del índice de probabilidad del diferencial de asignación de tratamiento debido a una covariable no observada" (DiPrete y Gangl, 2004). En el anexo A13 se calcula un nivel de significancia hipotético p-crítico para cada $\Gamma$ representando el límite del nivel significativo del efecto del tratamiento en el caso de autoselección endógena en el estatus de tratamiento. Este método provee la posibilidad de discernir la fuerza que los factores no observados necesitarían para hacer que el AтT sea resultado de un sesgo de selección.

La robustez a la posible presencia de sesgo de selección en un resultado puede ser determinada observando el nivel crítico de $\Gamma$ al que el $p$-crítico se vuelve significativo. Las variables se dicen ser sensibles a sesgo escondido si $\Gamma$ alcanza niveles estadísticamente significativos para valores por debajo de 2 . Sin embargo, un valor por debajo de 2, de 1.15 por ejemplo, no implica una falta de impacto del programa en la variable dependiente. En el anexo A13, para gasto en alimento este valor tendría que estar entre 2.4 y 2.6, lo que a su vez significa que el nivel crítico es logrado "si una covariable no observada causa que el índice de probabilidad de la asignación del tratamiento difiera entre caso de tratamiento y de control por un factor de (2.5) aproximadamente" o, en otras palabras, el sesgo escondido tendría que alcanzar valores para $\Gamma$ de entre 2.4 y
2.6 para poder declarar como espurio el impacto positivo de los créditos de FND en el gasto total en alimentos.

La mayoría de las variables presentadas tienen un valor significativo para $\Gamma$ por debajo de 2 . Sin embargo, hubo 11 variables para las que los valores críticos se ubicaron por arriba de 2, lo que significa que los resultados no son muy robustos a la selección de características no observables.

\section{Análisis prospectivo}

Los resultados obtenidos mostraron la capacidad de Financiera Nacional de Desarrollo Agropecuario, Rural, Forestal y Pesquero (FND) para impactar de manera positiva el nivel de vida de las familias beneficiarias por medio de las variables de salud, educación, alimentación e inversión. Este impacto fue posible pues FND contrarresta los efectos de las fallas en los mercados crediticios agrícolas en México que no permiten satisfacer la demanda de crédito en el sector rural. El importante rol económico que la agricultura juega en el Mexicali, y específicamente en su valle, vuelve crucial el acceso a mercados crediticios que permitan a los agricultores operar de manera eficiente y hacer inversiones que les permita mantenerse competitivos.

Sin embargo, cabe mencionar que FND tiene la oportunidad de tener un impacto aún más pronunciado en el bienestar de las familias beneficiarias por medio de información. Por ejemplo, FND podría brindar información a los beneficiarios de manera práctica por medio de folletos acerca de programas gubernamentales de salud, de alimentación saludable o consejos para mejorar los hábitos de lectura de los menores de edad, sólo por dar unos ejemplos. Este cambio operacional se podría combinar con otros organismos gubernamentales, especialmente los enfocados a zonas rurales para lograr una mayor eficacia.

Dado que los resultados mostrados son una clara evidencia del impacto de los créditos de la Financiera en las familias beneficiarias, se sugiere continuar, fortalecer y expandir el programa gubernamental de créditos. Siendo que la Financiera está logrando su objetivo de "desarrollar al medio rural a través de financiamiento en primer y segundo piso para cualquier actividad económica que se realice en poblaciones menores a 50000 habitantes resultando en la mejora de su calidad de vida" (FND, 2015), este artículo recomienda el reconocimiento de este organismo como medio para el desarrollo rural, que es donde se concentra la pobreza en México.

\section{Conclusiones y recomendaciones}

Una sugerencia para futuras investigaciones sobre el impacto de los créditos de FND es analizar también el impacto que los 
créditos otorgados por las instituciones intermediarias tienen en las familias de los productores. Además, se puede explorar la posibilidad de enfocarse solamente en el impacto de los créditos en una sola variable de bienestar, lo que permitiría obtener más entrevistas en el mismo tiempo al acortar el número de preguntas. Adicionalmente, dado que las observaciones de control fueron obtenidas a nivel nacional, se podría considerar la posibilidad de hacer las entrevistas en diversos estados, o procurar poblaciones donde haya mayor variación en el tamaño de los créditos y de esta manera permitir un análisis de impacto por tamaño de crédito. Como se discutió en capítulos anteriores, la calidad del matching podría ser mejorada con un mayor número de entrevistados. Adicionalmente, el matching podría ser mejor si se obtuvieran las observaciones de control de la misma comunidad de donde se obtengan las observaciones de tratamiento.
Estas sugerencias abordan la crítica a la validez de selección de los métodos de diseño por características observables, como el ppp y los métodos de regresión, pues si los individuos difieren en sus características no observables, los resultados no están exentos del sesgo. A pesar de que en este trabajo se hizo un esfuerzo por construir un vector de variables $X$ que incluyera todas las características que pudieran ser decisivas para que un individuo reciba el tratamiento o no, la probabilidad de controlar estas características si las observaciones del grupo de control y observaciones de tratamiento son obtenidas de la misma región serían mayores y es probable que más variables pasaran el análisis de sensibilidad ante la presencia de variables no observables con impacto en la selección al programa. Sin embargo, con el uso del test de sensibilidad se pudo concluir que la mayoría de los resultados eran robustos a la presencia de sesgo por características no observables.

Referencias

Azam, M., Ferré, C. y Ajwad, M. (2013). Can public works programs mitigate the impact of crises in Europe? The case of Latvia. IZA Journal of European Labor Studies, 2, 1-21.

Caliendo, M. y Kopeinig, S. (2005). Some practical guidance for the implementation of propensity score matching. Journal of Economic Surveys, 22(1): 31-72.

Clément, M. (2011). Remittances and household expenditure patterns in Tajikistan: A propensity score matching analysis. Asian Development Review, 28(2), 58-87.

Crépon, B., Devoto, F., Duflo, E. y Parienté, W. (2011). Impact of microcredit in rural areas of Morocco. Working Paper. International Growth Center. London School of Economic and Political Science.

Dehejia, R. H. y Wahba, S. (2002). Propensity score-matching methods for nonexperimental causal studies. Review of Economics and statistics, 84(1), 151-161.

DiPrete, T. A. y Gangl M. (2004). Assessing bias in the estimation of causal effects: Rosenbaum bounds on matching estimators and instrumental variables estimation with imperfect instruments. Sociological Methodology, 34(1), 271-310.

Encuesta Nacional sobre Niveles de Vida de los Hogares (ENNViH) (2012). ENNViH-3 2009 2012. Bases Nivel Hogar. Disponible en http://www.ennvih-mxfls.org/ennhiv-3.html. De Janvry, A., Dustan, A. y Sadoulet, E. (2011). Recent advances in impact analysis methods for ex-post impact assessments of agricultural technology: Options for the CGIAR. In Increasing the rigor of ex-post impact assessment of agricultural research: $A$ discussion on estimating treatment effects. 2 de octubre de 2010. University of California-Berkeley.

Financiera Nacional de Desarrollo Agropecuario, Rural, Forestal y Pesquero (2015). Misión y Visión. http://www.fnd.gob.mx/fr/ Paginas/MisionVision.aspx.

Liu, X. y Lynch, L. (2011). Do agricultural land preservation programs reduce farmland loss? Evidence from a propensity scorematching estimator. Land Economics, 87(2), 183-201.

Meng, X., Gong, X. y Wang, Y. (2009). Impact of income growth and economic reform on nutrition availability in urban
China: 1986-2000. Economic, Development and Cultural Change, 57(2), 261-295.

Pérez, C. M. A., Altamirano Cárdenas, R. y Rojas Herrera, J. J. (2009). Retos del crédito agrícola: estudio de caso de la intermediación financiera en el sur de Sonora, México. Región y sociedad, 21(46), $53-78$.

Pitt, M. M., Khandker, S. R., Chowdhury, O. H. y Millimet, D. L. (2003). Credit programs for the poor and the health status of children in rural Bangladesh. In ternational Economic Review, 44(1): 87-118.

Rosenbaum, P. R. (2002). Observational Studies (2nd ed.) New York: Springer.

Ruiz-Arranz, M., Davis, B., Handa, S., Stampini, M. y Winters, P. (2002). Program conditionality and food security: The impact of Progresa and Procampo transfers in rural Mexico. Revista Economia, $7(2): 249-278$

Soto Ibáñez, M.C. (2008). Financiera rural, influencia en dispersoras e intermediarios financieros rurales, zona centro del estado de Veracruz (tesis doctoral). México: Universidad Autónoma de México. 
Anexos

Anexo A1.

Diferencia en covariables antes del pareamiento.

\begin{tabular}{|c|c|c|c|c|c|c|}
\hline \multirow{2}{*}{ Factor } & \multicolumn{3}{|c|}{ Lista $\mathrm{A}^{\dagger}$} & \multicolumn{3}{|c|}{ Lista Bף } \\
\hline & Grupo 1 & Grupo 2 & Trat $=$ Contr & Grupo 1 & Grupo 2 & Trat $=$ Contr \\
\hline Número de cuartos usados para dormir & 2.127 & 3.700 & $* * *$ & 2.127 & 3.700 & $* * *$ \\
\hline Vivienda es propiedad propia & 0.783 & 0.950 & * & 0.783 & 0.950 & * \\
\hline Edad de el/la jefe/a de familia & 46.903 & 51.475 & N. S. & 46.903 & 51.475 & N. S. \\
\hline Educación de el/la jefe/a de familia & 3.837 & 6.600 & $* * *$ & 3.837 & 6.600 & $* * *$ \\
\hline Tamaño del hogar & 4.910 & 3.825 & $* *$ & 4.910 & 3.825 & $* *$ \\
\hline Hogar vive en zona urbana & 1.516 & 1.725 & $* *$ & 1.516 & 1.725 & $* *$ \\
\hline Hogar posee vacas y/o toros & 0.044 & 0.200 & $* * *$ & 0.044 & 0.200 & $* * *$ \\
\hline Hogar posee caballos o mulas & 0.050 & 0.250 & $* * *$ & 0.050 & 0.250 & $* * *$ \\
\hline Hogar posee cerdos o chivos & 0.053 & 0.100 & N.S. & 0.053 & 0.100 & N. S. \\
\hline Hogar posee gallinas o guajolotes & 0.117 & 0.200 & $*$ & 0.117 & 0.200 & * \\
\hline Hectáreas cultivadas en propieda privada & 1.518 & 7.205 & $* * *$ & 1.518 & 7.205 & $* * *$ \\
\hline Porcentaje de miembros del hogar de 0-6 años & 0.111 & 0.065 & * & 0.111 & 0.065 & * \\
\hline Porcentaje de miembros del hogar de 7-17 años & 0.152 & 0.107 & N. S. & 0.152 & 0.107 & N. S. \\
\hline Porcentaje de miembros del hogar de 18-24 años & 0.135 & 0.126 & N. S. & 0.135 & 0.126 & N. S. \\
\hline Porcentaje de miembros del hogar de 25-29 años & 0.086 & 0.160 & $* * *$ & 0.086 & 0.160 & $* * *$ \\
\hline Porcentaje de miembros del hogar de 30-39 años & 0.119 & 0.065 & * & 0.119 & 0.065 & * \\
\hline Porcentaje de miembros del hogar de 40-49 años & 0.172 & 0.283 & $* * *$ & 0.172 & 0.283 & $* * *$ \\
\hline Nivel de educación promedio de los miembros hombres del hogar & 4.294 & 7.038 & $* * *$ & 4.294 & 7.038 & $* * *$ \\
\hline Hogar cuenta con cuenta de banco & 0.236 & 0.825 & $* * *$ & 0.236 & 0.825 & $* * *$ \\
\hline Hectáreas cultivadas en ejido & 2.670 & 13.925 & $* * *$ & $-\cdot$ & $-\cdot$ & $-\cdot$ \\
\hline Número de mujeres en el hogar & 2.275 & 2.025 & N. S. & -- & -- & -- \\
\hline Hectárease cultivadas por el hogar & 9.251 & 71.525 & $* * *$ & -- & -- & $-\cdot$ \\
\hline Porcentaje de mujeres en el hogar & -- & -- & -- & 0.468 & 0.497 & N. S. \\
\hline Ingreso anual del hogar & -- & -- & -- & 184545 & 234214 & N. S. \\
\hline
\end{tabular}

Notas: ${ }^{\dagger} t$-test de covariables para análisis de alimentación, salud e inversión. ${ }^{\uparrow} t$-test de covariables para análisis de educación. N. S. = no significativo y significativo; *, **, *** $=$ significativo a un $p \geq 0.05,0.01$ o 0.001 , respectivamente. Trat $=$ tratamiento. Contr $=$ control.

Fuente: elaboración propia con datos obtenidos de la encuesta realizada para la presente investigación y ENNviH (2012).

Anexo A2. Probit para calibrar el puntaje de propensión.

\begin{tabular}{|c|c|c|c|c|}
\hline \multirow{2}{*}{ Variable } & \multicolumn{2}{|c|}{ Lista $\mathrm{A}^{\dagger}$} & \multicolumn{2}{|c|}{ Lista B } \\
\hline & Coeficiente & Error Estándar & Coeficiente & Error Estándar \\
\hline Número de cuartos para dormir & 0.087 & 0.042 & 0.089 & 0.039 \\
\hline Vivienda es propiedad propia & 0.782 & 0.617 & 0.321 & 0.467 \\
\hline Edad de el/la jefe/a de familia & -0.005 & 0.012 & -0.002 & 0.010 \\
\hline Edución de el/la jefe/a de familia & 0.098 & 0.086 & 0.095 & 0.077 \\
\hline Tamaño del hogar & -0.506 & 0.155 & -0.295 & 0.089 \\
\hline Hogar vive en zona urbana & -0.019 & 0.310 & 0.221 & 0.294 \\
\hline Hogar posee vacas y/o toros & 0.029 & 0.343 & 0.015 & 0.298 \\
\hline Hogar posee caballos o mulas & -0.009 & 0.350 & 0.133 & 0.305 \\
\hline Hogar posee cerdos o chivos & -0.320 & 0.438 & -0.187 & 0.383 \\
\hline Hogar posee gallinas o guajolotes & 0.328 & 0.312 & 0.251 & 0.278 \\
\hline hectareas cultivadas enpropiedad privada & 0.024 & 0.011 & 0.024 & 0.010 \\
\hline Porcentaje miembros del hogar 0-6 años & -0.424 & 1.182 & -1.133 & 1.101 \\
\hline Porcentaje miembros del hogar 7-17 años & 0.332 & 0.943 & 0.329 & 0.850 \\
\hline Porcentaje miembros del hogar 18-24 años & 0.075 & 0.931 & -0.113 & 0.828 \\
\hline Porcentaje miembros del hogar 25-29 años & 1.235 & 0.901 & 1.086 & 0.757 \\
\hline Porcentaje miembros del hogar 30-39 años & -1.173 & 0.953 & -1.023 & 0.840 \\
\hline Porcentaje miembros del hogar 40-49 años & -0.115 & 0.544 & -0.113 & 0.495 \\
\hline Nivel de educación promedio de los miembros hombres del hogar & 0.136 & 0.093 & 0.176 & 0.086 \\
\hline Cuenta de banco & 0.917 & 0.260 & 0.945 & 0.242 \\
\hline Hectáreas cultivadas en ejido & 0.026 & 0.009 & -- & -- \\
\hline Número de mujeres en el hogar & 0.392 & 0.191 & -- & -- \\
\hline Hectáreas cultivadas por el hogar & 0.010 & 0.003 & -- & -- \\
\hline Porcentaje de mujeres en el hogar & -- & -- & 1.298 & 0.631 \\
\hline Ingreso anual del hogar & -- & -- & 0.000 & 0.000 \\
\hline Constate & -3.269 & 1.198 & -3.959 & 1.092 \\
\hline Pseudo $R^{2}$ & \multicolumn{2}{|c|}{0.5833} & \multicolumn{2}{|c|}{0.4921} \\
\hline LR chi $^{2}$ & \multicolumn{2}{|c|}{203.42} & \multicolumn{2}{|c|}{172.19} \\
\hline$p>\operatorname{chi}^{2}$ & \multicolumn{2}{|c|}{0.0000} & \multicolumn{2}{|c|}{0.0000} \\
\hline Número de observaciones & \multicolumn{2}{|c|}{1274} & \multicolumn{2}{|c|}{1293} \\
\hline $\begin{array}{l}\text { Notas: }{ }^{\dagger} t \text {-test de covariables para análisis de alimentación, salud e inversión } \\
\text { significativo a un } p \geq 0.05,0.01 \text { o } 0.001 \text {, respectivamente. } \\
\text { Fuente: elaboración propia con datos obtenidos de la encuesta realizada para }\end{array}$ & est de covaria & análisis de educac & $=$ no significa & ficativo; *, **, *** \\
\hline
\end{tabular}




\begin{tabular}{|c|c|c|c|c|c|c|}
\hline \multirow{2}{*}{ Variable } & \multicolumn{3}{|c|}{ Lista $\mathrm{A} 1 \S$} & \multicolumn{3}{|c|}{ Lista $\mathrm{A} 2^{\Phi}$} \\
\hline & Tratamiento & Control & Trat $=$ Contr & Tratamiento & Control & Trat $=$ Contr \\
\hline Número de cuartos usados para dormir & 3.647 & 2.853 & * & 3.407 & 2.630 & * \\
\hline Vivienda es propiedad propia & 0.971 & 0.941 & N. S. & 0.963 & 0.926 & N. S. \\
\hline Edad de el/la jefe/a de familia & 52.971 & 53.824 & N. S. & 51.148 & 52.741 & N. S. \\
\hline Educación de el/la jefe/a de familia & 6.441 & 7.000 & N. S. & 6.000 & 6.482 & N. S. \\
\hline Tamaño del hogar & 3.971 & 4.559 & N. S. & 4.000 & 4.444 & N. S. \\
\hline Hectáreas cultivadas por el hogar & 34.412 & 51.971 & N. S. & 29.852 & 50.964 & N. S. \\
\hline Hogar vive en zona urbana & 1.677 & 1.618 & N. S. & 1.667 & 1.704 & N. S. \\
\hline Hogar posee vacas y/o toros & 0.176 & 0.147 & N. S. & 0.185 & 0.185 & N. S. \\
\hline Hogar posee caballos o mulas & 0.265 & 0.382 & N. S. & 0.259 & 0.296 & N. S. \\
\hline Hogar posee cerdos o chivos & 0.118 & 0.059 & N. S. & 0.111 & 0.074 & N. S. \\
\hline Hogar posee gallinas o guajolotes & 0.206 & 0.118 & N. S. & 0.222 & 0.148 & N. S. \\
\hline Hectáreas cultivadas en ejido & 11.647 & 9.486 & N. S. & 8.630 & 7.649 & N. S. \\
\hline Hectáreas cultivadas en propiedad privada & 8.265 & 12.485 & N. S. & 7.407 & 5.537 & N. S. \\
\hline Número de mujeres en el hogar & 2.118 & 2.618 & N. S. & 2.074 & 2.333 & N. S. \\
\hline Porcentaje de miembros del hogar de 0-6 años & 0.076 & 0.111 & N. S. & 0.088 & 0.103 & N. S. \\
\hline Porcentaje de miembros del hogar de 7-17 años & 0.112 & 0.129 & N. S. & 0.120 & 0.119 & N. S. \\
\hline Porcentaje de miembros del hogar de 18-24 años & 0.135 & 0.124 & N. S. & 0.114 & 0.120 & N. S. \\
\hline Porcentaje de miembros del hogar de 25-29 años & 0.138 & 0.160 & N. S. & 0.139 & 0.164 & N. S. \\
\hline Porcentaje de miembros del hogar de 30-39 años & 0.047 & 0.066 & N. S. & 0.052 & 0.084 & N. S. \\
\hline Porcentaje de miembros del hogar de 40-49 años & 0.270 & 0.219 & N. S. & 0.273 & 0.209 & N. S. \\
\hline Nivel de educación promedio de los miembros hombres del hogar & 6.9129 & 7.1961 & N. S. & 6.3718 & 6.7284 & N. S. \\
\hline Hogar cuenta con cuenta de banco & 0.79412 & 0.73529 & N. S. & 0.74074 & 0.74074 & N. S. \\
\hline Pseudo $R^{2}$ & & 0.256 & & & 0.287 & \\
\hline LR chi $^{2}$ & & 24.100 & & & 21.500 & \\
\hline$p>\operatorname{chi}^{2}$ & & 0.342 & & & 0.490 & \\
\hline
\end{tabular}

Anexo A4. Impacto del Tratamiento sobre los Tratados (ATT) de FND en el gasto en alimentos.

\begin{tabular}{|c|c|c|c|c|c|c|c|c|}
\hline & \multicolumn{4}{|c|}{ Lista $\mathrm{A} 1 \S$} & \multicolumn{4}{|c|}{ Lista $\mathrm{A} 2^{\Phi}$} \\
\hline & Tratamiento & Control & Diferencia & Estad. T & Tratamiento & Control & Diferencia & Estad. T \\
\hline & \multicolumn{4}{|c|}{ Gasto total per cápita en } & \multicolumn{4}{|c|}{ Porcentaje del gasto total en alimentos en } \\
\hline Carne & 92.32 & 25.77 & 66.56 & 6.08 & 0.284 & 0.143 & 0.141 & 4.53 \\
\hline Bienes inferiores & 32.63 & 15.65 & 16.98 & 2.11 & 0.099 & 0.085 & 0.014 & 0.80 \\
\hline Frutas y vegetales & 111.50 & 22.76 & 88.74 & 5.41 & 0.304 & 0.150 & 0.155 & 5.61 \\
\hline Alimentos industrializados & 41.30 & 8.69 & 32.61 & 5.11 & 0.146 & 0.039 & 0.107 & 5.00 \\
\hline Refrescos & 23.96 & 6.04 & 17.91 & 4.31 & 0.080 & 0.029 & 0.051 & 3.20 \\
\hline Tortillas & 22.08 & 7.81 & 14.27 & 4.31 & 0.067 & 0.042 & 0.025 & 1.85 \\
\hline Alimentos & 341.89 & 170.73 & 171.15 & 3.35 & -- & -- & -- & $-\cdot$ \\
\hline
\end{tabular}


Anexo A5. Impacto del Tratamiento sobre los Tratados (ATT) de FND en diferentes categorías de gasto de alimentos per cápita, métodos alternativos de pareamiento (matching).

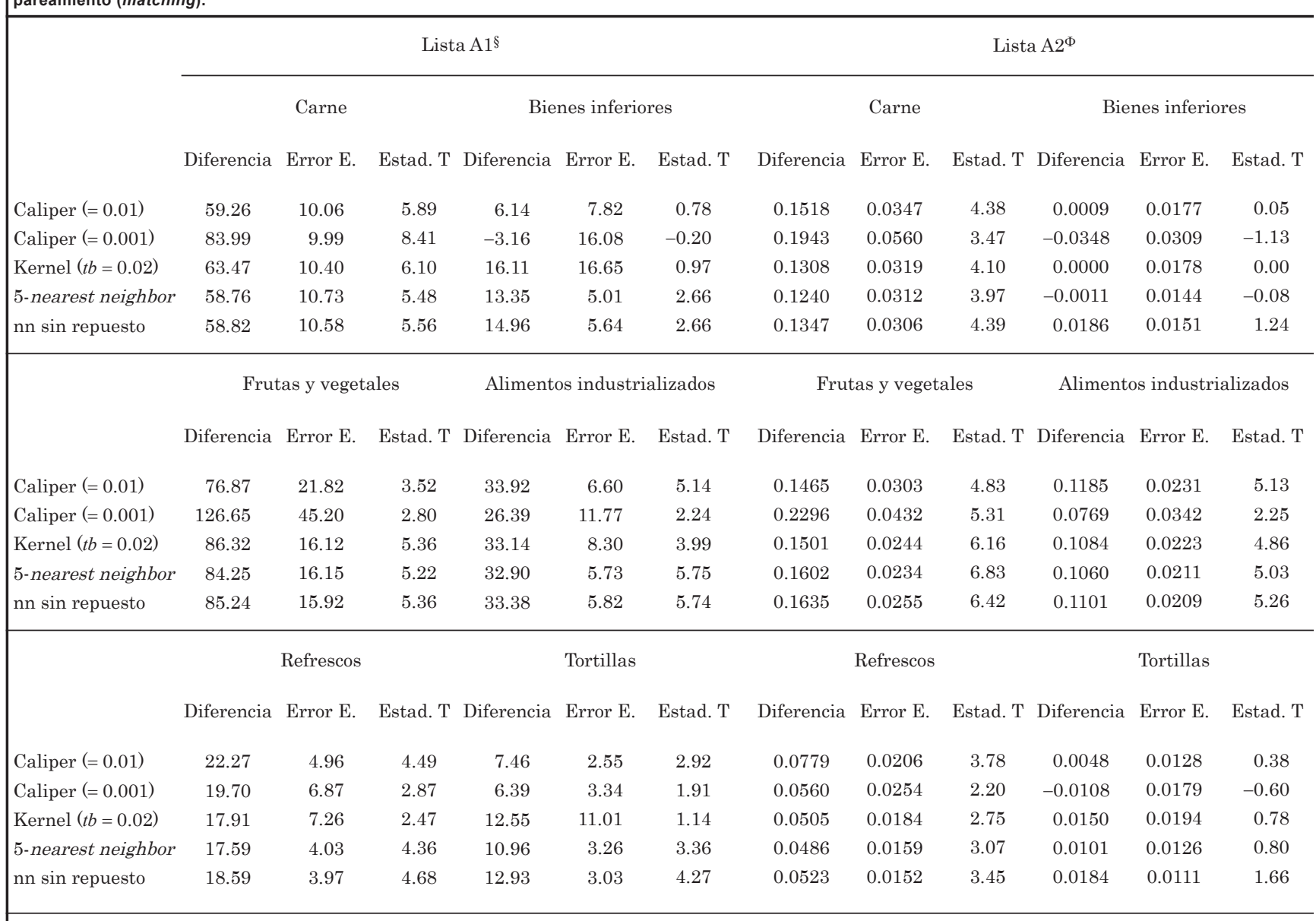

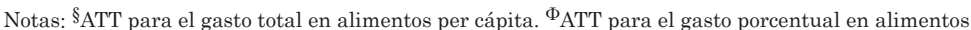

Fuente: elaboración propia con datos obtenidos de la encuesta realizada para la presente investigación y ENNViH (2012).

Anexo A6. Impacto del Tratamiento sobre los Tratados (ATT) de FND en inversión.

\begin{tabular}{lccc} 
& Tratamiento & Control & Diferencia \\
\hline Posesión de un tractor & 0.8824 & 0.4412 & 0.4412 \\
Piso de madera o loseta & 0.7353 & 0.6765 & 0.0588 \\
\hline Fuente: elaboración propia con datos obtenidos de la encuesta realizada para la presente investigación y ENNviH (2012). & 0.42 \\
\hline
\end{tabular}

Anexo A7. ATT de FND en diferentes categorías de inversión. Métodos alternativos de pareamiento (matching).

Tractor

Diferencia

Caliper $(=0.01)$

Caliper $(=0.001)$

Kernel $(t b=0.02)$

5-nearest neighbor

$\mathrm{nn}$ sin repuesto

\begin{abstract}
0.3810
0.3333

0.5838

0.6824

0.6176
\end{abstract}

Error Estándar
0.1445
0.2079
0.0815
0.0886
0.0951

Estadístico T

2.64
1.6
7.16
7.7
6.49

Piso de madera/loseta

Diferencia
0.0000
-0.2222
0.1130
0.1353
0.0588

Error Estándar

0.1580

0.2291

0.1192

0.1143

0.1119
Estadístico T

$$
\begin{array}{r}
-0.97 \\
0.95 \\
1.18
\end{array}
$$

0.53

Nota: $\mathrm{nn}$ sin repuesto $=$ nearest neighbor sin repuesto.

Fuente: elaboración propia con datos obtenidos de la encuesta realizada para la presente investigación y ENNviH (2012). 
Anexo A8. Diferencia en covariables después del pareamiento para el gasto en alimentos per cápita.

\begin{tabular}{|c|c|c|c|c|c|c|}
\hline \multirow{2}{*}{ Variable } & \multicolumn{3}{|c|}{ Lista $\mathrm{B} 1^{\dagger \dagger}$} & \multicolumn{3}{|c|}{ Lista B2ף } \\
\hline & Trat. & Contr. & Trat $=$ Contr & Tratamiento & Contr. & Trat $=$ Contr \\
\hline Ingreso anual del hogar & $2.50 \mathrm{E}+05$ & $5.60 \mathrm{E}+05$ & N. S. & 260000.00 & 380000.00 & N. S. \\
\hline Número de cuartos usados para dormir & 3.8846 & 5.5 & N. S. & 3.82 & 5.95 & N. S. \\
\hline Vivienda es propiedad propia & 1 & 0.96154 & N. S. & 1.00 & 0.95 & N. S. \\
\hline Edad de el/la jefe/a de familia & 50.462 & 46.154 & N. S. & 48.46 & 44.77 & N. S. \\
\hline Educación de el/la jefe/a de familia & 7.0385 & 7 & N. S. & 7.05 & 6.91 & N. S. \\
\hline Tamaño del hogar & 4.3846 & 5.0769 & N. S. & 4.50 & 4.91 & N. S. \\
\hline Hogar vive en zona urbana & 1.6154 & 1.6923 & N. S. & 1.64 & 1.68 & N. S. \\
\hline Hogar posee vacas y/o toros & 0.15385 & 0.15385 & N. S. & 0.18 & 0.14 & N. S. \\
\hline Hogar posee caballos o mulas & 0.26923 & 0.11538 & N. S. & 0.32 & 0.09 & N. S. \\
\hline Hogar posee cerdos o chivos & 0.15385 & 0.07692 & N. S. & 0.14 & 0.09 & N. S. \\
\hline Hogar posee gallinas o guajolotes & 0.15385 & 0.03846 & N. S. & 0.18 & 0.05 & N. S. \\
\hline Hectáreas cultivadas en prop. Privada & 10.346 & 7.6187 & N. S. & 10.50 & 5.73 & N. S. \\
\hline Porcentaje de mujeres en el hogar & 0.51987 & 0.49258 & N. S. & 0.52 & 0.47 & N. S. \\
\hline Porcentaje de miembros del hogar de 0-6 años & 0.09936 & 0.11868 & N. S. & 0.11 & 0.12 & N. S. \\
\hline Porcentaje de miembros del hogar de 7-17 años & 0.15449 & 0.12253 & N. S. & 0.16 & 0.13 & N. S. \\
\hline Porcentaje de miembros del hogar de 18-24 años & 0.12692 & 0.16529 & N. S. & 0.15 & 0.17 & N. S. \\
\hline Porcentaje de miembros del hogar de 25-29 años & 0.17115 & 0.15119 & N. S. & 0.14 & 0.16 & N. S. \\
\hline Porcentaje de miembros del hogar de 30-39 años & 0.06154 & 0.08581 & N. S. & 0.07 & 0.09 & N. S. \\
\hline Porcentaje de miembros del hogar de 40-49 años & 0.23718 & 0.23535 & N. S. & 0.25 & 0.24 & N. S. \\
\hline Nivel de educación promedio de los miembros hombres del hogar & 7.42 & 7.26 & N. S. & 7.18 & 7.15 & N. S. \\
\hline Hogar cuenta con cuenta de banco & 0.76923 & 0.92308 & N. S. & 0.77 & 0.91 & N. S. \\
\hline Pseudo $R^{2}$ & & 0.332 & & & 0.296 & \\
\hline LR chi $^{2}$ & & 23.49 & & & 17.64 & \\
\hline$p>\operatorname{chi}^{2}$ & & 0.265 & & & 0.611 & \\
\hline \multicolumn{7}{|c|}{ 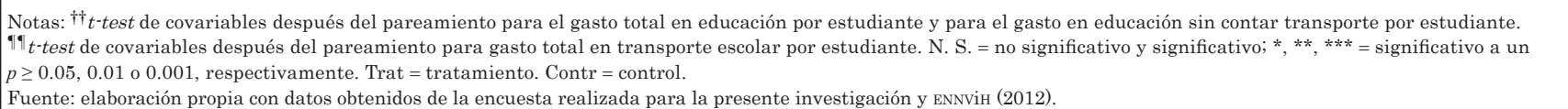 } \\
\hline
\end{tabular}

Anexo A9.

Impacto del Tratamiento sobre los Tratados (ATT) de FND en educación.

\begin{tabular}{|c|c|c|c|c|}
\hline & Tratamiento & Control & Diferencia & Estad. T \\
\hline Gasto total educativo por estudiante & 5519.94 & 715.00 & 4804.94 & 3.97 \\
\hline Gasto educativo por estudiante excluyendo transporte & 4624.36 & 641.92 & 3982.44 & 3.81 \\
\hline Gasto en transporte escolar por estudiante & 1058.41 & 86.36 & 972.05 & 3.06 \\
\hline
\end{tabular}

Anexo A10. Impacto del Tratamiento sobre los Tratados (ATT) de FND en gastos educativos por estudiante. Métodos alternativos de pareamiento (matching).

\begin{tabular}{|c|c|c|c|c|c|c|c|c|c|}
\hline & \multicolumn{3}{|c|}{ Gasto total educativo } & \multicolumn{3}{|c|}{ Gasto total exclendo transporte } & \multicolumn{3}{|c|}{ Gasto en transporte escolar } \\
\hline & Diferencia & Error E. & Estad. T & Diferencia & Error E. & Estad. T & Diferencia & Error E. & Estad. T \\
\hline Caliper $(=0.01)$ & 4263.4 & 1421.3 & 3 & 3241.8 & 1126.10 & 2.88 & 1094.64 & 459.68 & 2.38 \\
\hline Caliper $(=0.001)$ & 3023.1 & 1424.4 & 2.12 & 2476.3 & 1215.40 & 2.04 & 546.88 & 282.96 & 1.93 \\
\hline Kernel $(t b=0.02)$ & 4356.7 & 1254.0 & 3.47 & 3633.3 & 1091.63 & 3.33 & 857.83 & 320.45 & 2.68 \\
\hline 5-nearest neighbor & 3870.7 & 1368.1 & 2.83 & 3120.8 & 1228.37 & 2.54 & 913.50 & 321.59 & 2.84 \\
\hline nn $\sin$ repuesto & 4563.0 & 1193.2 & 3.82 & 3709.7 & 1030.13 & 3.6 & 1008.41 & 314.22 & 3.21 \\
\hline
\end{tabular}


Anexo A11. Impacto del Tratamiento sobre los Tratados (ATT) de FND en salud.

\begin{tabular}{|c|c|c|c|c|c|c|c|c|}
\hline & $\begin{array}{r}\text { Tratamiento } \\
\text { Total }\end{array}$ & $\begin{array}{l}\text { Control } \\
\text { visitas }\end{array}$ & Diferencia & Estad. T & $\begin{array}{l}\text { Tratamiento } \\
\text { Porcenta }\end{array}$ & $\begin{array}{l}\text { Control } \\
\text { de indivi }\end{array}$ & $\begin{array}{l}\text { Diferencia } \\
\text { os en el hoga }\end{array}$ & Estad. T \\
\hline $\begin{array}{l}\text { Número de individuos que enfermaron y } \\
\text { visitaron un doctor }\end{array}$ & 0.35 & 0.29 & 0.06 & 0.27 & 0.1221 & 0.0569 & 0.0651 & 1.20 \\
\hline $\begin{array}{l}\text { Número de individuos que enfermaron y } \\
\text { no visitaron un doctor }\end{array}$ & 0.09 & 1.53 & -1.44 & -4.86 & 0.0162 & 0.3603 & -0.3441 & -5.45 \\
\hline $\begin{array}{l}\text { Número de individuos que no } \\
\text { enfermaron y visitaron un doctor }\end{array}$ & 0.41 & 0.00 & 0.41 & 3.94 & 0.1132 & 0.0000 & 0.1132 & 3.23 \\
\hline $\begin{array}{l}\text { Número de individuos que no } \\
\text { enfermaron y no visitaron un doctor }\end{array}$ & 3.12 & 2.74 & 0.38 & 0.82 & 0.7485 & 0.5828 & 0.1658 & 2.11 \\
\hline Número de individuos que enfermaron & 0.44 & 1.82 & -1.38 & -4.40 & 0.1382 & 0.4172 & -0.2790 & -3.83 \\
\hline $\begin{array}{l}\text { Número de individuos que visitaron un } \\
\text { doctor }\end{array}$ & 0.76 & 0.29 & 0.47 & 2.00 & 0.2353 & 0.0569 & 0.1784 & 2.86 \\
\hline
\end{tabular}

Anexo A12.

Impacto del Tratamiento sobre los Tratados (ATT) de FND en diferentes categorías de salud, métodos alternativos de pareamiento (matching).

ATT en el número de individuos

ATT en el porcentaje de individuos

\begin{tabular}{cccc}
\hline $\begin{array}{c}\text { Enfermos y que visitaron } \\
\text { un doctor }\end{array}$ & $\begin{array}{c}\text { Enfermos y no visitaron un } \\
\text { doctor }\end{array}$ & $\begin{array}{c}\text { ATT en el porcentaje de } \\
\text { individuos que enfermaron y }\end{array}$ & ATT en el porcentaje de \\
& & visitaron un doctor & no visitaron un doctor
\end{tabular}

Diferencia Error E. Estad. T Diferencia Error E. Estad. T Diferencia Error E. Estad. T Diferencia Error E. Estad. T

\begin{tabular}{|c|c|c|c|c|c|c|c|c|c|c|c|c|}
\hline Caliper $(=0.01)$ & -0.10 & 0.22 & -0.43 & -1.67 & 0.31 & -5.39 & 0.0356 & 0.0646 & 0.55 & -0.4000 & 0.0660 & -6.06 \\
\hline Caliper $(=0.001)$ & -0.11 & 0.28 & -0.40 & -1.44 & 0.52 & -2.77 & 0.0386 & 0.0726 & 0.53 & -0.3278 & 0.1043 & -3.14 \\
\hline Kernel $(t b=0.02)$ & 0.09 & 0.17 & 0.54 & -1.39 & 0.28 & -5.02 & 0.0647 & 0.0517 & 1.25 & -0.3357 & 0.0528 & -6.36 \\
\hline 5-nearest neighbor & 0.08 & 0.17 & 0.46 & -1.57 & 0.23 & -6.91 & 0.0571 & 0.0504 & 1.13 & -0.3750 & 0.0483 & -7.76 \\
\hline \multirow[t]{3}{*}{$\mathrm{nn} \sin$ repuesto } & 0.00 & 0.16 & 0.00 & -1.59 & 0.23 & -6.86 & 0.0472 & 0.0483 & 0.98 & -0.3887 & 0.0488 & -7.96 \\
\hline & \multicolumn{3}{|c|}{$\begin{array}{c}\text { No enfermaron y visitaron } \\
\text { un doctor }\end{array}$} & \multicolumn{3}{|c|}{$\begin{array}{l}\text { No enfermaron y no visitaron } \\
\text { un doctor }\end{array}$} & \multicolumn{3}{|c|}{$\begin{array}{c}\text { No enfermaron y visitaron } \\
\text { un doctor }\end{array}$} & \multicolumn{3}{|c|}{$\begin{array}{c}\text { No enfermaron y no visitaron } \\
\text { un doctor }\end{array}$} \\
\hline & Diferencia & Error E. & Estad. T & Diferencia & Error E. & Estad. T & Diferencia & Error E. & Estad. T & Diferencia & Error E. & Estad. T \\
\hline Caliper $(=0.01)$ & 0.38 & 0.13 & 2.96 & 0.67 & 0.54 & 1.23 & 0.1151 & 0.0511 & 2.25 & 0.2493 & 0.0877 & 2.84 \\
\hline Caliper $(=0.001)$ & 0.33 & 0.17 & 2.00 & 0.22 & 0.92 & 0.24 & 0.0759 & 0.0428 & 1.78 & 0.2132 & 0.1050 & 2.03 \\
\hline Kernel $(t b=0.02)$ & 0.37 & 0.12 & 3.13 & 0.51 & 0.59 & 0.87 & 0.0937 & 0.0379 & 2.47 & 0.1772 & 0.0744 & 2.38 \\
\hline 5-nearest neighbor & 0.36 & 0.11 & 3.18 & 0.90 & 0.38 & 2.38 & 0.0848 & 0.0443 & 1.91 & 0.2331 & 0.0690 & 3.38 \\
\hline nn $\sin$ repuesto & 0.41 & 0.10 & 3.94 & 0.91 & 0.38 & 2.38 & 0.1132 & 0.0350 & 3.23 & 0.2282 & 0.0690 & 3.31 \\
\hline
\end{tabular}

Enfermaron

Visitaron un doctor

Enfermaron

Visitaron un doctor

Diferencia Error E. Estad. T Diferencia Error E. Estad. T Diferencia Error E. Estad. T Diferencia Error E. Estad. T

\begin{tabular}{|c|c|c|c|c|c|c|c|c|c|c|c|c|}
\hline Caliper $(=0.01)$ & -1.76 & 0.31 & -5.61 & 0.29 & 0.24 & 1.21 & -0.3644 & 0.0800 & -4.55 & 0.1507 & 0.0744 & 2.03 \\
\hline Caliper $(=0.001)$ & -1.56 & 0.47 & -3.28 & 0.22 & 0.29 & 0.76 & -0.2892 & 0.1068 & -2.71 & 0.1146 & 0.0731 & 1.57 \\
\hline Kernel $(t b=0.02)$ & -1.30 & 0.31 & -4.17 & 0.46 & 0.21 & 2.25 & -0.2709 & 0.0681 & -3.98 & 0.1585 & 0.0616 & 2.57 \\
\hline 5-nearest neighbor & -1.49 & 0.26 & -5.84 & 0.44 & 0.19 & 2.27 & -0.3179 & 0.0648 & -4.90 & 0.1419 & 0.0631 & 2.25 \\
\hline nn $\sin$ repuesto & -1.59 & 0.26 & -6.05 & 0.41 & 0.19 & 2.18 & -0.3415 & 0.0621 & -5.50 & 0.1604 & 0.0571 & 2.81 \\
\hline
\end{tabular}

Fuente: elaboración propia con datos obtenidos de la encuesta realizada para la presente investigación y ENNviH (2012). 


\begin{tabular}{|c|c|c|c|c|c|c|c|c|c|c|c|}
\hline$\Gamma$ & 1 & 1.2 & 1.4 & 1.6 & 1.8 & 2 & 2.2 & 2.4 & 2.6 & 2.8 & 3 \\
\hline \multirow[b]{2}{*}{$P$-value } & \multicolumn{11}{|c|}{ Gasto total en comida ( $N=34$ pares emparejados $)$} \\
\hline & 0.000 & 0.001 & 0.002 & 0.006 & 0.011 & 0.020 & 0.031 & 0.046 & 0.063 & 0.082 & 0.104 \\
\hline \multirow[b]{2}{*}{$P$-value } & \multicolumn{11}{|c|}{ Gasto total en comida per cápita ( $N=34$ pares emparejados) } \\
\hline & 0.000 & 0.000 & 0.000 & 0.001 & 0.002 & 0.004 & 0.007 & 0.011 & 0.016 & 0.022 & 0.029 \\
\hline \multirow{3}{*}{$P$-value } & \multicolumn{11}{|c|}{ Gasto total en carne per cápita ( $N=34$ pares emparejados) } \\
\hline & 0.000 & 0.000 & 0.000 & 0.000 & 0.000 & 0.001 & 0.002 & 0.003 & 0.004 & 0.006 & 0.009 \\
\hline & \multicolumn{11}{|c|}{ Gasto total en bienes inferiores per cápita $(N=34$ pares emparejados $)$} \\
\hline \multirow[t]{2}{*}{$P$-value } & 0.000 & 0.000 & 0.001 & 0.002 & 0.004 & 0.007 & 0.012 & 0.019 & 0.027 & 0.036 & 0.047 \\
\hline & \multicolumn{11}{|c|}{ Gasto total en frutas y verduras per cápita ( $N=34$ pares emparejados) } \\
\hline \multirow[t]{2}{*}{$P$-value } & 0.000 & 0.000 & 0.000 & 0.000 & 0.000 & 0.000 & 0.000 & 0.001 & 0.001 & 0.002 & 0.002 \\
\hline & \multicolumn{11}{|c|}{ Gasto total en alimentos industrializados per cápita $(N=34$ pares emparejados $)$} \\
\hline \multirow[t]{2}{*}{$P$-value } & 0.000 & 0.000 & 0.000 & 0.001 & 0.001 & 0.003 & 0.005 & 0.007 & 0.011 & 0.015 & 0.021 \\
\hline & \multicolumn{11}{|c|}{ Gasto total en refrescos per cápita ( $N=34$ pares emparejados $)$} \\
\hline$P$-value & 0.000 & 0.000 & 0.001 & 0.002 & 0.004 & 0.007 & 0.012 & 0.018 & 0.026 & 0.036 & 0.047 \\
\hline & & & Porce & je del gas & alimen & stinado & irne $(N=$ & ares em & ados) & & \\
\hline$P$-value & 0.000 & 0.000 & 0.001 & 0.003 & 0.006 & 0.010 & 0.016 & 0.022 & 0.030 & 0.039 & 0.049 \\
\hline & & & rcentaje & gasto en & entos de & do a bie & inferiore & $=34$ par & iparejad & & \\
\hline$P$-value & 0.085 & 0.168 & 0.267 & 0.371 & 0.470 & 0.561 & 0.640 & 0.708 & 0.766 & 0.813 & 0.851 \\
\hline & & & Porcen & del gast & alimento & tinado & tillas $(N$ & pares en & ejados) & & \\
\hline$P$-value & 0.006 & 0.019 & 0.045 & 0.083 & 0.132 & 0.190 & 0.254 & 0.320 & 0.385 & 0.449 & 0.510 \\
\hline & & & centaje & gasto en & ntos de & lo a fru & vegetale & $=34 \mathrm{par}$ & nparejac & & \\
\hline$P$-value & 0.000 & 0.000 & 0.001 & 0.001 & 0.003 & 0.005 & 0.007 & 0.011 & 0.015 & 0.020 & 0.025 \\
\hline & & & aje del g & $o$ en alim & destina & aliment & dustrial & $\mathrm{s}(N=3$ & es empa & & \\
\hline$P$-value & 0.000 & 0.000 & 0.001 & 0.002 & 0.004 & 0.007 & 0.011 & 0.016 & 0.022 & 0.029 & 0.037 \\
\hline & & & Porcen & del gastc & limento & tinado & $\operatorname{escos}(N$ & pares en & ejados） & & \\
\hline$P$-value & 0.001 & 0.004 & 0.011 & 0.023 & 0.042 & 0.066 & 0.096 & 0.131 & 0.170 & 0.211 & 0.254 \\
\hline & & & & & ón de tr & $(N=34$ & s empar & & & & \\
\hline$P$-value & 0.000 & 0.001 & 0.003 & 0.006 & 0.011 & 0.017 & 0.026 & 0.035 & 0.047 & 0.059 & 0.073 \\
\hline & & & Mate & 1 del piso & viviend & nadera & $\operatorname{eta}(N=$ & ares emp & ados) & & \\
\hline$P$-value & 0.264 & 0.365 & 0.457 & 0.540 & 0.611 & 0.673 & 0.725 & 0.769 & 0.806 & 0.837 & 0.863 \\
\hline & & & & Gasto tota & cativo $\mathrm{p}$ & tudiant & $=26$ par & parejad & & & \\
\hline$P$-value & 0.000 & 0.001 & 0.002 & 0.004 & 0.007 & 0.011 & 0.016 & 0.022 & 0.029 & 0.038 & 0.047 \\
\hline & & & Gasto & cativo po & adiante & yendo $t$ & oorte $(\mathrm{N}$ & pares en & ejados) & & \\
\hline$P$-value & 0.002 & 0.006 & 0.013 & 0.024 & 0.039 & 0.058 & 0.080 & 0.105 & 0.131 & 0.159 & 0.188 \\
\hline & & & & o en tran & e escola & estudia & $(N=22 \mathrm{r}$ & empare & & & \\
\hline$P$-value & 0.002 & 0.004 & 0.009 & 0.015 & 0.024 & 0.033 & 0.044 & 0.056 & 0.069 & 0.083 & 0.097 \\
\hline & & & úmero d & dividuos & enferma & visitar & doctorr & 34 pare & parejado & & \\
\hline$P$-value & 0.207 & 0.302 & 0.393 & 0.477 & 0.552 & 0.618 & 0.675 & 0.724 & 0.766 & 0.802 & 0.832 \\
\hline & & & imero de & lividuos c & afermar & o visite & un docto & $=34$ par & iparejad & & \\
\hline$P$-value & 0.000 & 0.000 & 0.000 & 0.000 & 0.000 & 0.000 & 0.000 & 0.000 & 0.000 & 0.000 & 0.000 \\
\hline & & & imero de & lividuos o & o enfern & $\mathrm{y}$ visite & un docto & $=34$ par & aparejad & & \\
\hline$P$-value & 0.000 & 0.001 & 0.002 & 0.003 & 0.005 & 0.007 & 0.010 & 0.013 & 0.016 & 0.020 & 0.023 \\
\hline & & & hero de $\mathrm{i}$ & viduos qu & enferms & no visi & un doct & $V=34 \mathrm{pa}$ & mpareja & & \\
\hline$P$-value & 0.178 & 0.317 & 0.461 & 0.589 & 0.696 & 0.780 & 0.843 & 0.890 & 0.923 & 0.947 & 0.963 \\
\hline & & & & ímero de & iduos qu & ermaro & $=34 \mathrm{pa}$ & mpareja & & & \\
\hline$P$-value & 0.000 & 0.000 & 0.000 & 0.000 & 0.000 & 0.000 & 0.000 & 0.000 & 0.000 & 0.000 & 0.000 \\
\hline & & & & ro de ind & os que & on un & $r(N=34$ & s empar & & & \\
\hline$P$-value & 0.002 & 0.005 & 0.012 & 0.024 & 0.040 & 0.060 & 0.084 & 0.111 & 0.141 & 0.172 & 0.205 \\
\hline & & & ccentaje & individuo & enferm & $y$ visita & in doctor & $=34 \mathrm{par}$ & nparejac & & \\
\hline$P$-value & 0.148 & 0.227 & 0.308 & 0.386 & 0.458 & 0.523 & 0.582 & 0.635 & 0.681 & 0.722 & 0.757 \\
\hline & & & centaje d & adividuos & enferma & no visi & un doct & $=34 \mathrm{pa}$ & mpareja & & \\
\hline$P$-value & 0.000 & 0.000 & 0.000 & 0.000 & 0.000 & 0.000 & 0.000 & 0.000 & 0.000 & 0.000 & 0.000 \\
\hline & & & centaje $d$ & adividuos & no enfer & $\mathrm{n}$ y visi & un doct & $I=34 \mathrm{pa}$ & mpareja & & \\
\hline$P$-value & 0.000 & 0.001 & 0.002 & 0.003 & 0.005 & 0.007 & 0.010 & 0.013 & 0.016 & 0.020 & 0.024 \\
\hline & & & ntaje de & lividuos $\mathrm{q}$ & enferm & y no vis & on un do & $N=34 \mathrm{p}$ & empare & & \\
\hline$P$-value & 0.004 & 0.015 & 0.036 & 0.068 & 0.111 & 0.162 & 0.219 & 0.280 & 0.341 & 0.402 & 0.461 \\
\hline & & & & centaje do & viduos & ffermar & $N=34 \mathrm{p}$ & emparej & & & \\
\hline$P$-value & 0.000 & 0.000 & 0.000 & 0.000 & 0.000 & 0.000 & 0.000 & 0.000 & 0.000 & 0.000 & 0.000 \\
\hline & & & Por & taje de in & uos que & aron un & or $(N=3$ & ces empa & dos) & & \\
\hline$P$-value & 0.001 & 0.003 & 0.006 & 0.013 & 0.022 & 0.035 & 0.050 & 0.067 & 0.087 & 0.108 & 0.131 \\
\hline
\end{tabular}

